\title{
Iterated-map approach to die tossing
}

\section{Feldberg, Rasmus; Szymkat, Maciej; Knudsen, Carsten; Mosekilde, Erik}

\section{Published in:}

Physical Review A

Link to article, DOI:

10.1103/PhysRevA.42.4493

Publication date:

1990

Document Version

Publisher's PDF, also known as Version of record

Link back to DTU Orbit

Citation (APA):

Feldberg, R., Szymkat, M., Knudsen, C., \& Mosekilde, E. (1990). Iterated-map approach to die tossing. Physical Review A, 42(8), 4493-4502. https://doi.org/10.1103/PhysRevA.42.4493

\section{General rights}

Copyright and moral rights for the publications made accessible in the public portal are retained by the authors and/or other copyright owners and it is a condition of accessing publications that users recognise and abide by the legal requirements associated with these rights.

- Users may download and print one copy of any publication from the public portal for the purpose of private study or research.

- You may not further distribute the material or use it for any profit-making activity or commercial gain

- You may freely distribute the URL identifying the publication in the public portal

If you believe that this document breaches copyright please contact us providing details, and we will remove access to the work immediately and investigate your claim. 


\title{
Iterated-map approach to die tossing
}

\author{
Rasmus Feldberg, Maciej Szymkat, ${ }^{*}$ Carsten Knudsen, and Erik Mosekilde \\ Physics Laboratory III, The Technical University of Denmark, DK-2800 Lyngby, Denmark
}

(Received 25 April 1990)

\begin{abstract}
Nonlinear dissipative mapping is applied to determine the trajectory of a two-dimensional die thrown onto an elastic table. The basins of attraction for different outcomes are obtained and their distribution in the space of initial conditions discussed. The system has certain properties in common with chaotic systems. However, a die falls to rest after a finite number of impacts, and therefore the system has a finite sensitivity to the initial conditions. Quantitative measures of this sensitivity are proposed and their variations with the initial momentum and orientation of the die investigated.
\end{abstract}

\section{INTRODUCTION}

The discovery of chaos ${ }^{1,2}$ has shown that unpredictability can perfectly well occur in deterministic dynamical systems. One type of system, which at first sight may appear to possess this property, is that of die tossing. By tradition, this system is considered an ideal mechanism for producing random outcomes. Usually, the motion of a die is so rapid that the human eye is unable to follow the trajectory in detail. Combined with the actual complexity of the motion, which apparently had defied mathematical treatment for centuries, this may have contributed to the widespread notion that a die toss is random.

However, by virtue of the dissipation of energy, a die must always come to rest within a finite time. This leaves little hope for the kind of unpredictability observed in chaotic systems or in systems with fractal basin boundaries. These phenomena are related to motions that can be sustained forever. For dissipative systems this can occur only if there is an external forcing or another form of more or less continuous energy supply. Even the possibility of a chaotic transient ${ }^{3,4}$ appears to be ruled out by these arguments, since this type of dynamics implies a finite probability of sustaining the motion for arbitrarily long times. Therefore we must conclude that a die toss cannot show the sensitive dependence on initial conditions found in chaotic systems. Nonetheless, die tossing has certain properties in common with these systems. In particular, the basins of attraction in the space of initial conditions show a certain recurrence in structure.

In the present paper we shall investigate the structure of these basins of attraction by means of a recursive transformation involving the construction of flight and collision maps. A somewhat similar approach has been applied to coin tossing by Vulovic and Prange. ${ }^{5}$ Besides treating a different problem, we provide a much more detailed interpretation of the variation in outcome. A preliminary discussion of this variation was given by Knudsen et $a l .{ }^{6}$ and by Mosekilde et al. ${ }^{7}$. These results were based on time-continuous simulations of the complete trajectory, involving slightly different assumptions about the impact process. In particular, the collision time was considered to be finite, allowing a certain rotation of the die during a collision. In the limit of short collision times, the two approaches give identical results. For a more comprehensive study, the mapping approach is advantageous because it requires orders of magnitude less computer time.

In an actual toss, the initial conditions of the die are reproducible with finite accuracy only. If the initial energy is sufficiently small, it is possible to throw the die such that the outcome is certain. Only if the die has energy enough to roll several times, the outcome appears to be random. But how much is this energy, and how random is the outcome? It is clearly of interest to determine the sensitivity of the outcome to variations in the initial conditions. For chaotic systems, perturbations of the initial conditions grow exponentially with time, making it impossible to predict the behavior beyond a certain time horizon. ${ }^{8}$ With a more precise specification of the initial conditions, this horizon is extended, but even the smallest inaccuracy will sooner or later limit the predictability. For a die toss, on the other hand, inaccuracies in the initial conditions are amplified only through a finite number of collision-flight processes. Thus, although the basins of attraction for different outcomes may be rather complicated, they are finite in size and are bounded by regular surfaces.

We propose quantitative measures for the average size of the basins of attraction and for the mixing of these basins. Thereafter, we apply these measures to determine how the sensitivity to the initial conditions varies with the energy and orientation of the die.

\section{FLIGHT MAP}

Let us consider the trajectory of a die thrown onto the surface of an ideally flat table. The die has mass $m$, sidelength $a$, and moment of inertia $I=\frac{1}{6} m a^{2}$. For simplicity, the die is restricted to move in the vertical $x y$ plane with a pair of sides parallel to this plane. The motion may then be described in terms of six dynamical variables: the horizontal position of the center of mass $x$, the vertical position of this point $y$, and the orientation of the die $\varphi$, together with their conjugated momentum coordi- 
nates $p_{x}, p_{y}$, and $L . \varphi$ is defined as the angle between a particular side of the die and the horizontal direction. The table is represented by the plane $y=0$. Because of the perfect translational symmetry of the problem, the value of $x$ is immaterial, and this variable can be left out of the calculations.

The motion consists of free flights interrupted by collisions between die and table. Both of these processes may be represented by a deterministic nonlinear map, and the total trajectory can be constructed by iterating these maps until the die has lost sufficient energy for the final outcome to be determined. Because of the constraint between $y$ and $\varphi$ that exists in the moment of collision, this approach justifies neglecting $y$ as a variable. Thus the flight map $F$ transforms the dynamic variables at the beginning of a flight $\left(p_{x}^{*}, p_{y}^{*}, \varphi^{*}, L^{*}\right)$ into the post-flight values $\left(p_{x}, p_{y}, \varphi, L\right)$. Similarly, the collision map $C$ relates the dynamic variables after a collision to their pre-collision values, i.e., $C:\left(p_{x}, p_{y}, \varphi, L\right)$ $\rightarrow\left(p_{x}^{*}, p_{y}^{*}, \varphi^{*}, L^{*}\right)$. The asterisk is used to indicate postcollision, pre-flight values.

During a flight, only the force of gravity acts upon the die. This implies that $p_{x}$ and $L$ remain constant. Moreover, the dynamics of the flight are completely integrable. Thus we immediately have the flight map $F$ :

$$
\begin{aligned}
& p_{x}=p_{x}^{*}, \\
& p_{y}=p_{y}^{*}-m g t, \\
& \varphi=\varphi^{*}+\frac{L^{*}}{I} t, \\
& L=L^{*},
\end{aligned}
$$

where $g$ is the gravitational acceleration and $t$ is the time of flight.

To determine $t$ we use

$$
\frac{a}{\sqrt{2}} \cos \left(\widetilde{\varphi}-\frac{\pi}{4}\right)=\frac{a}{\sqrt{2}} \cos \left(\widetilde{\varphi}^{*}-\frac{\pi}{4}\right)+\frac{p_{y}^{*}}{m} t-\frac{1}{2} g t^{2} .
$$

Here a tilde denotes mod $(\pi / 2)$. Equation (5) expresses the condition of contact between die and table, and the smallest strictly positive solution to $(5)$ is to be inserted into (2) and (3) to determine the post-flight values of $p_{y}$ and $\varphi$. The only difficulty lies in a proper root bracketing.

Besides regular flights in which the die starts out with a positive vertical momentum and ends with a negative $p_{y}$, Eqs. (1)-(4) encompass two particular kinds of flight: (a) flights initiated with a negative $p_{y}$, and (b) flights initiated with a positive $p_{y}$ and terminated while $p_{y}$ is still positive. Such flights are possible provided that the angular momentum $L$ is large enough for the vertical velocity of the lowest corner to have the opposite direction of the center-of-mass velocity $p_{y} / m$. They will be referred to as short flights. An important property of short flights is that the outcome cannot change because the maximum altitude gained in such a flight is insufficient for a corner to pass under the center of mass.

\section{COLLISION MAP}

In order to obtain the collision map $C$ we make the following conjectures concerning the impacts between die and table.

Conjecture 1. The collision time is negligible short.

This implies that the orientation of the die $\varphi$ remains constant during the collision. Derivation of $C$ is hereby reduced to finding expressions for $p_{x}^{*}, p_{y}^{*}$, and $L^{*}$ in terms of $p_{x}, p_{y}, \varphi$, and $L$.

Conjecture 2. The vertical velocity of a colliding die corner is changed such that the ratio of the post- and pre-impact values remain constant:

$$
v_{c y}^{*}=-\beta v_{c y} .
$$

Although a rigorous physical justification for this conjecture may be hard to provide, it appears to be a standard assumption for collisions between rigid bodies. ${ }^{9}$

The value of $\beta$ depends upon the properties of the colliding bodies. We suppose that plastic deformation and permanent damage to either table or die can be neglected. In a simple model, the table reaction in the vertical direction will then consist of an elastic term with damping. If the impact between a die corner and the table is described as half a period of a damped harmonic oscillation, $\beta$ can be expressed as

$$
\beta=\exp \left[-\pi \alpha /\left(4 m k-\alpha^{2}\right)^{1 / 2}\right] .
$$

Here $\alpha$ is the damping coefficient for the table reaction, and $k$ is the elastic force constant. For a particular combination of die and table, $\beta$ can be estimated such that the typical number of impacts before the die comes to rest is correct. As previously noted, a real toss is too swift for us to follow it with the eye. The ear appears to have a somewhat better temporal resolution. By replaying simulations of a toss in real time, and by coding the computer to emit a sound for each collision, this can be used to adjust $\beta$ until the computer simulation sounds like a real toss. $^{7}$ In the calculations to be presented here we have taken $\beta=0.50$.

Conjecture 3. Due to frictional forces, the horizontal velocity of the corner in contact with the table is usually brought to zero during the impact, i.e.,

$$
v_{c x}^{*}=0 \text {. }
$$

Simulations of a continuous version of the system with a more detailed representation of the collision forces have shown ${ }^{7}$ that this will be the case in many impacts. However, frictional forces are not always sufficient to avoid sliding collisions in which the horizontal velocity of the corner in contact with the table remains nonzero.

Assuming that the frictional force is determined by the normal force times the friction coefficient $\mu$, as a complement to $(8)$ we have the following.

Conjecture 4. The ratio of the horizontal to the normal changes in momentum can never exceed the friction coefficient.

Thus, if (8) produces a result that is in violation of conjec- 
ture $4,(8)$ is replaced by

$$
\left|p_{x}^{*}-p_{x}\right|=\mu\left|p_{y}^{*}-p_{y}\right|,
$$

where the sign of the horizontal momentum change is chosen to be opposite to the sign of the horizontal velocity for the sliding corner $v_{c x}$. Hereafter, collisions in which the frictional forces are large enough to stop the horizontal motion of the colliding corner will be referred to as nonsliding collisions.

Combining the above conjectures, one obtains the following relations, which form the basis for calculations of the collision map for nonsliding collisions:

$$
\begin{aligned}
& \frac{p_{x}^{*}}{m}+\frac{a L^{*}}{\sqrt{2} I} \cos \left(\widetilde{\varphi}-\frac{\pi}{4}\right)=0, \\
& \frac{p_{y}^{*}}{m}-\frac{a L^{*}}{\sqrt{2} I} \cos \left(\tilde{\varphi}+\frac{\pi}{4}\right)=-\beta\left[\frac{p_{y}}{m}-\frac{a L}{\sqrt{2} I} \cos \left[\widetilde{\varphi}+\frac{\pi}{4}\right)\right],
\end{aligned}
$$

and

$$
\begin{aligned}
L^{*}-L= & \frac{a}{\sqrt{2}}\left(p_{x}^{*}-p_{x}\right) \cos \left[\widetilde{\varphi}-\frac{\pi}{4}\right] \\
& -\frac{a}{\sqrt{2}}\left(p_{y}^{*}-p_{y}\right) \cos \left(\widetilde{\varphi}+\frac{\pi}{4}\right) .
\end{aligned}
$$

As previously defined $\widetilde{\varphi}=\varphi \bmod \pi / 2$, and $\beta$ is given by (7). The first two equations merely represent a transformation of (8) and (6) into the center-of-mass coordinates. Equation (12) expresses the change in angular momentum in terms of the corresponding changes in the linear momenta.

Introducing

$$
\begin{aligned}
\Delta L=-\frac{m}{4} \frac{a}{\sqrt{2}}[ & v_{c x} \cos \left[\tilde{\varphi}-\frac{\pi}{4}\right] \\
& \left.-(1+\beta) v_{c y} \cos \left(\tilde{\varphi}+\frac{\pi}{4}\right]\right],
\end{aligned}
$$

with

$$
v_{c x}=\frac{p_{x}}{m}+\frac{a L}{\sqrt{2} I} \cos \left(\widetilde{\varphi}-\frac{\pi}{4}\right)
$$

and

$$
v_{c y}=\frac{p_{y}}{m}-\frac{a L}{\sqrt{2} I} \cos \left(\widetilde{\varphi}+\frac{\pi}{4}\right),
$$

the above set of three coupled linear equations (10)-(12) are readily solved to give

$$
\begin{aligned}
& p_{x}^{*}=p_{x}-m v_{c x}-\frac{a m}{\sqrt{2} I} \Delta L \cos \left(\tilde{\varphi}-\frac{\pi}{4}\right), \\
& p_{y}^{*}=p_{y}-(1+\beta) m v_{c y}+\frac{a m}{\sqrt{2} I} \Delta L \cos \left(\widetilde{\varphi}+\frac{\pi}{4}\right), \\
& \varphi^{*}=\varphi
\end{aligned}
$$

$$
L^{*}=L+\Delta L
$$

These equations define the collision map for nonsliding collisions $C_{\mathrm{ns}}$.

If the change in $p_{x}$ calculated from the above equations is in violation of conjecture 4 , then (8) is replaced by (9) to produce the following collision map for sliding collisions $C_{\mathrm{S}}$ :

$p_{x}^{*}=p_{x} \pm \mu\left[(1+\beta) m v_{c y}-\frac{a m}{\sqrt{2} I} \Delta L \cos \left[\widetilde{\varphi}+\frac{\pi}{4}\right]\right]$,

$p_{y}^{*}=p_{y}-(1+\beta) m v_{c y}+\frac{a m}{\sqrt{2} I} \Delta L \cos \left(\tilde{\varphi}+\frac{\pi}{4}\right)$,

$\varphi^{*}=\varphi$,

$L^{*}=L+\Delta L$

with

$$
\Delta L=\frac{(1+\beta) m v_{c y} \frac{a}{\sqrt{2}}\left[\cos \left(\tilde{\varphi}+\frac{\pi}{4}\right) \pm \mu \cos \left(\tilde{\varphi}-\frac{\pi}{4}\right)\right]}{1 \pm 3 \mu \cos \left(\tilde{\varphi}-\frac{\pi}{4}\right) \cos \left(\tilde{\varphi}+\frac{\pi}{4}\right)+3 \cos ^{2}\left[\tilde{\varphi}+\frac{\pi}{4}\right)} .
$$

As before $v_{c y}$ is given by (15). The sign of the terms involving $\mu$ is chosen to be opposite the sign of $v_{c x}$. In combination, $C_{\mathrm{ns}}$ and $C_{\mathrm{S}}$ define the overall collision map C.

It sometimes happens, particularly towards the end of the trajectory, that the die starts on a series of very rapid collision-flight pairs involving the same corner. This signals the beginning of a continuous sliding process in which a corner of the die remains in contact with the table surface, as it move along it. Our iteration scheme is not well suited for this type of collision since they lead to a large number of iterations with small changes in the dynamical variables. Instead, when these collisions occur, the map is replaced by a set of differential equations, which describe the continuous sliding process:

$$
\begin{aligned}
& \dot{p}_{x}= \pm \mu F_{n}, \\
& \dot{p}_{y}=F_{n}-m g, \\
& \dot{\varphi}=\frac{L}{I},
\end{aligned}
$$

and

$$
\dot{L}=-\frac{a}{\sqrt{2}} F_{n}\left[\cos \left[\widetilde{\varphi}+\frac{\pi}{4}\right] \pm \mu \cos \left[\widetilde{\varphi}-\frac{\pi}{4}\right]\right] .
$$

Here

$$
F_{n}=\frac{m\left[g-\frac{a}{\sqrt{2}}\left(\frac{L}{I}\right]^{2} \cos \left(\tilde{\varphi}-\frac{\pi}{4}\right)\right]}{1 \pm 3 \mu \cos \left[\widetilde{\varphi}-\frac{\pi}{4}\right] \cos \left[\widetilde{\varphi}+\frac{\pi}{4}\right]+3 \cos ^{2}\left[\widetilde{\varphi}+\frac{\pi}{4}\right]}
$$


is the force required to keep $v_{c y}=0$. The integration procedure is stopped if a neighboring corner comes in contact with the table, if the remaining energy becomes too low for a change in the outcome to occur, or when $F_{n}$ ceases to be positive.

\section{DISTRIBUTION OF PREIMAGES}

By iterating the above flight and collision maps we can follow the motion of the die through a complete toss. This motion is simply interpreted as a series of collisionflight pairs. After a certain number of such pairs, the energy of the die has fallen below the value

$$
E_{\mathrm{cr}}=m g a / \sqrt{2} \text {, }
$$

and the outcome can no longer change. At this time the iteration is stopped. The critical energy $E_{\mathrm{cr}}$ is the potential energy of a die balancing directly on one of its edges.

In this section we shall investigate the distribution of basins of attraction (or preimages) for the various outcomes. We shall concentrate on mapping out the variation of the outcome with the initial values of $\varphi$ and $p_{y}$, taking the initial values of $p_{x}$ and $L$ to be zero. In this way we restrict our investigation to a subset of the total initial condition space. However, the qualitative distribution of the basins of attraction in this space seems to be generic. This means that changes in the initial values of $p_{x}$ and $L$ only produce smooth transformations of the basins of attraction. A few results will be shown for $p_{x 0} \neq 0$.

Thus, in the following calculation, each toss is initiated with a collision in which the die has a (negative) vertical momentum $p_{y 0}$ and an orientation $\varphi_{0}$. This corresponds to the situation after a vertical drop of the die from an altitude

$$
h_{0}=\frac{p_{y 0}^{2}}{2 m^{2} g}+\frac{a}{\sqrt{2}} \cos \left(\varphi_{0}-\frac{\pi}{4}\right) .
$$

Figure 1 shows the variation in outcome after the first collision-flight pair, i.e., after a single iteration of $F \circ C$, with $\circ$ denoting functional composition. This outcome is defined as the side of the die which is up by the time the die terminates the flight. The horizontal axis represents the initial orientation of the die, and the vertical axis measures the initial momentum in terms of $m \sqrt{g a}$. With the grey-tone code used to designate the various outcomes, black denotes 1 , dark grey denotes 2 , light grey 3 , and white 4.

After the first collision-flight pair, the distribution of outcomes is still quite simple. In fact, this distribution can easily be derived analytically. ${ }^{10}$ On the left-hand side of the figure, corresponding to initial angles $\varphi_{0}<0.25$ rad, the die lands relatively flat on one of its sides. Under these conditions, the die suffers a considerable change in its angular momentum, and its starts to rotate quite rapidly. At first sight one might suppose that this would lead to a complex distribution of outcomes. However, a large angular momentum after a flat collision implies that a neighboring corner will collide with the table, before the die really takes off. Thus the flight will be short, and

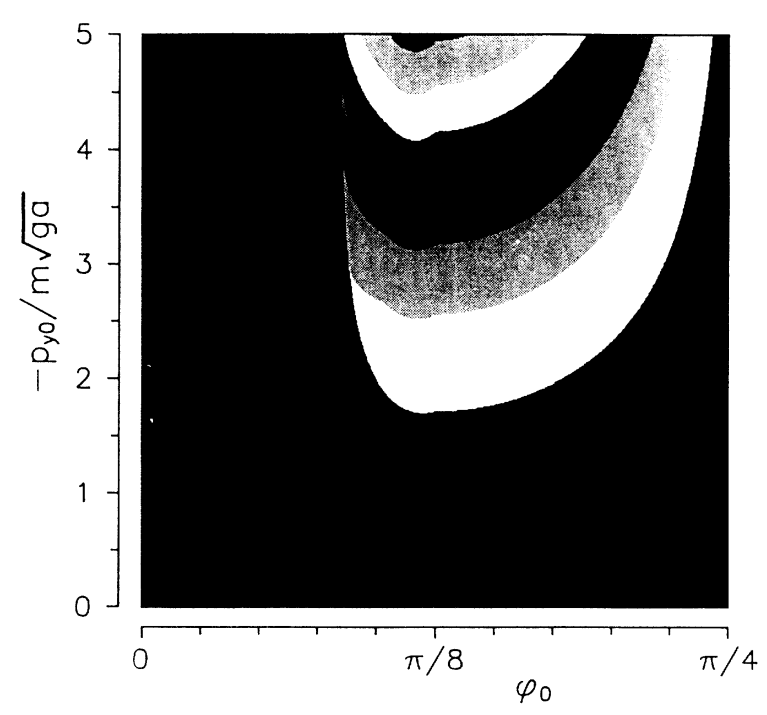

FIG. 1. Outcome distribution after one iteration of the combined collision-flight map $F \circ C$. The horizontal axis represents the orientation of the die before the first collision, and the vertical axis shows the normalized initial momentum $p_{y 0} / m \sqrt{g a}$. The die is assumed to fall vertically from an altitude $h_{0}=p_{y 0}^{2} / 2 m^{2} g+(a / \sqrt{2}) \cos \left(\varphi_{0}-\pi / 4\right)$. With the applied color code, black represents 1 , dark grey 2 , light grey 3 , and white 4 . The lack of structure on the left-hand side of the figure is due to short flights. Note the discontinuity in the outcome variation across the borderline between short and long flights.

it does not lead to a change in outcome. Our analytical calculations confirm that the borderline for short flights falls on top of the curve separating the black region of constant outcome to the left in Fig. 1 from the zone with varying outcome.

The outmost right-hand side of Fig. 1 corresponds to tosses in which the die falls almost directly on one of its

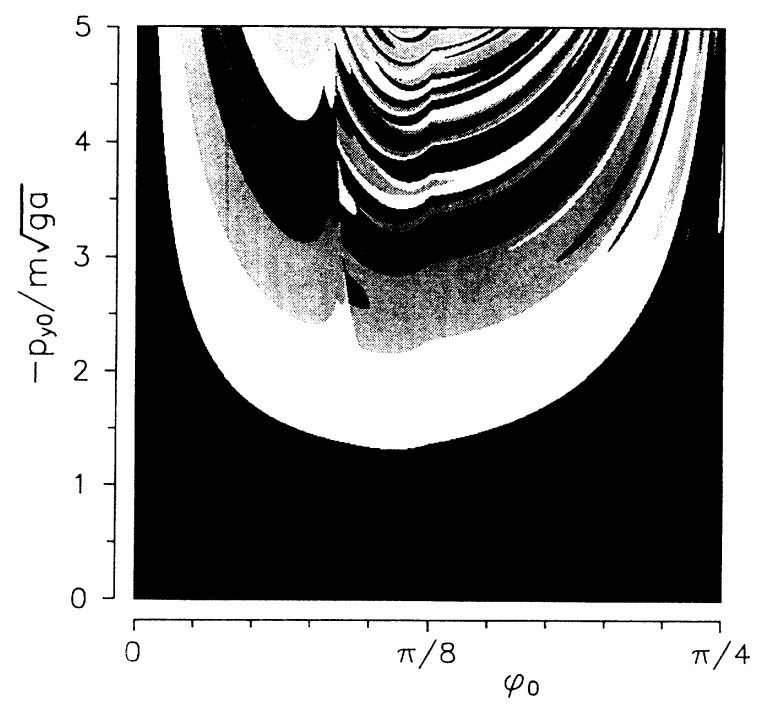

FIG. 2. Outcome distribution after two iterations of $F \circ C$. The second collision gives rise to a continuation of the outcome distribution produced by the first impact into the left-hand side of the space of initial conditions. Broadly speaking, this continuation has the form of a slightly deformed mirror image of the structure on the right-hand side of Fig. 1. On the righthand side of this figure, additional structure has now emerged in each of the previous bands of constant outcome. 
edges. In this case, the center of mass is right above the point of collision, and the die suffers only a small change in its angular momentum. Thus the narrow black region of outcome 1 stretching along the right edge of Fig. 1 corresponds to flights in which the die rotates so slowly that it maintains the same side up, until the next collision. As the angle $\varphi_{0}$ is decreased, the gain in angular momentum in the collision increases, and the die rotates faster and faster in the subsequent flight. This explains the curtainlike structure of bands with constant outcome. It is observed that these follow in a regular order. As one would expect, the number of possible changes in outcome during the first collision-flight process increases with increasing initial momentum. Between these bands and the region of constant outcome to the left there is an essential discontinuity in the outcome variation. On one side of the discontinuity, the die is caught by the impact of a neighboring corner, on the other side, the die can fly and rotate as long as its energy permits. This essential discontinuity is not lifted by the subsequent iterations of $F \circ C$.

Near the middle of the plot, one observes a vertically running series of break points in the borderlines between the various bands. These break points lie on the threshold between sliding and nonsliding collisions, with sliding collisions to the left. This is also directly confirmed by analytical calculations.

By iterating $F \circ C$ a second time, we find the outcome distribution after two collision-flight pairs. As illustrated in Fig. 2, this distribution is considerably more complex than the previous distribution. Nonetheless, we can still understand the main elements of the structure.

On the left-hand side of Fig. 2 we observe a curtainlike structure similar to the structure observed on the righthand side of Fig. 1. We recall that the lack of structure in the left-hand side of that figure was due to short flights. In such a flight, a neighboring corner collides with the table almost immediately after the takeoff from the first collision. Broadly speaking, the second collision produces a continuation of the outcome distribution of Fig. 1 into the right-hand side of the outcome space. Because of the additional losses in energy associated with the second impact, this continuation is shifted to somewhat higher initial momenta, and the discontinuity in outcome variation produced by the first collision is not completely repaired. Otherwise, the continuation has the form of a slightly deformed mirror image of the outcome distribution in Fig. 1. In particular, we observe the series of break points separating sliding and nonsliding collisions, now with the sliding collisions to the right. Thus, to the left of the borderline for short flights in the first impact, the outcome structure is delayed one iteration of F。C

On the right-hand side of Fig. 2, additional structure has developed in each of the former bands of constant outcome. In particular, we observe how tongues penetrate into these bands from the top and from the borderline between short and long flights. Similar tongues can be observed in the outcome distribution for coin tossing. ${ }^{5}$ Each band in Fig. 1 represents a variation of the pre-collision angle $\varphi_{0}$ for the second impact over the in- terval from $-\pi / 4$ to $\pi / 4$. This is twice the angular variation considered for the first impact in Fig. 1. If this variation was extended to $-\pi / 4$, we would see the mirror image of Fig. 1, only with the bands following in the opposite order. Therefore one would expect that each band from Fig. 1 now contains a substructure consisting of a double version of the outcome distribution in Fig. 1, only raised to higher initial energies.

This conjecture is readily verified. Thus, in Fig. 3(a) we have magnified and transformed part of one of the bands in Fig. 2 into a rectangular shape similar to the shape of Fig. 1. One immediately sees the resemblance in structure between the two figures. Even the borderlines between sliding and nonsliding collisions are clearly visible. This recursive property is reminiscent of the selfsimilar structure of fractal basin boundaries found in many nonlinear dynamical systems. ${ }^{11,12}$ However, if we wanted to trace a structure by repetitive magnifications of parts of the final outcome distribution, we would have to let $p_{y 0}$ increase for each iteration. This is in contrast to fractal basin boundaries where the repetitions of struc-
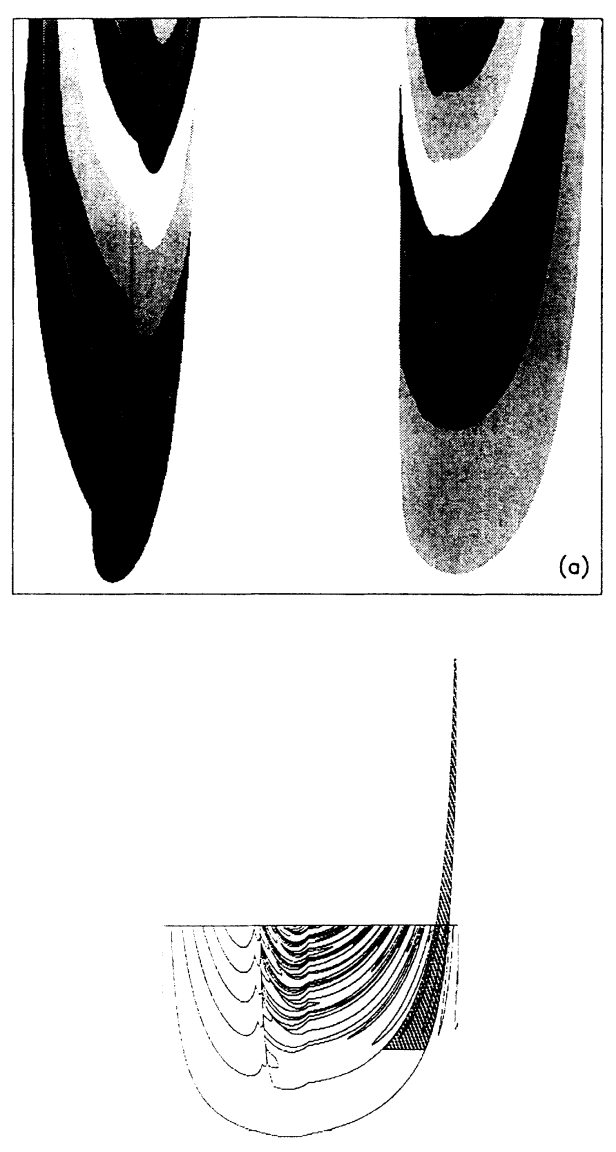

(b)

FIG. 3. Amplification and transformation of one of the bands in Fig. 2 show that the substructure produced by the second impact contains a double version of the structure observed in Fig. 1 after the first collision. This is expressive of the recurrent properties of the mapping process. The sketch in (b) shows the part of Fig. 2 that has been transformed into (a). 
ture lie within the structure itself.

It is worth noticing that, like the outcome distribution in Fig. 1, the substructure which has emerged in the bands of Fig. 2 contains lines of discontinuity in the outcome variation. These lines arise from the boundary between long and short flights after the second impact. In this way, discontinuity in the outcome variation spreads over the distribution.

After three iterations of $F \circ C$, the outcome distribution is more complicated. This is illustrated in Fig. 4. However, the qualitative changes that occur from Fig. 2 to Fig. 4 are merely a repetition of the transformation that leads from Fig. 1 to Fig. 2. The substructure already observed on the right-hand side of Fig. 2 now appears on the left-hand side of Fig. 4. On the right-hand side of Fig. 4, many of the former areas of constant outcome have been divided into several smaller areas, and again there is a certain self-similarity in the process. In the lower part of the plot, where the initial energy of the die is relatively small, the change in outcome distribution is only quantitative.

Further applications of $F \circ C$ introduce additional structure in the outcome distribution. The upper part becomes more complicated as each of the former regions of constant outcome splits into several, but not infinitely many, smaller areas. After a number of iterations, the energy has fallen below $E_{\mathrm{cr}}$ for all tosses within the considered region of the space of initial conditions. From then on, further iterations no longer change the outcome distribution. Figure 5 shows an example of such a final outcome distribution. It is interesting to note that even for relatively high initial momenta, one can still find regions where the initial conditions can be varied significantly without changing the outcome.

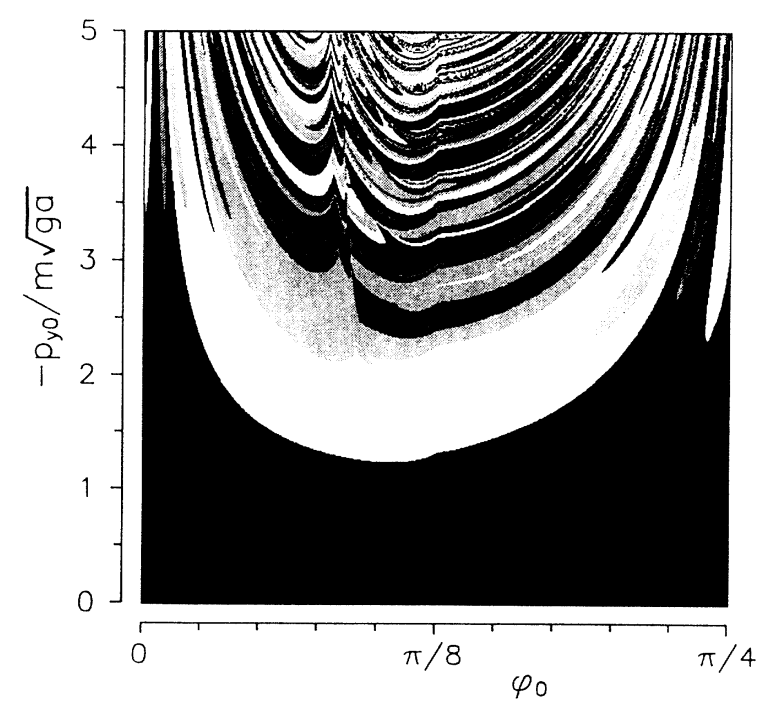

FIG. 4. Outcome distribution after three iterations of $F \circ C$. The substructure observed on the right-hand side of Fig. 2 now appears on the left-hand side of this figure. On the right-hand side, additional substructure has appeared, as many of the areas of constant outcome in Fig. 2 have been divided into several smaller ones. Again there is a certain self-similarity in the process.

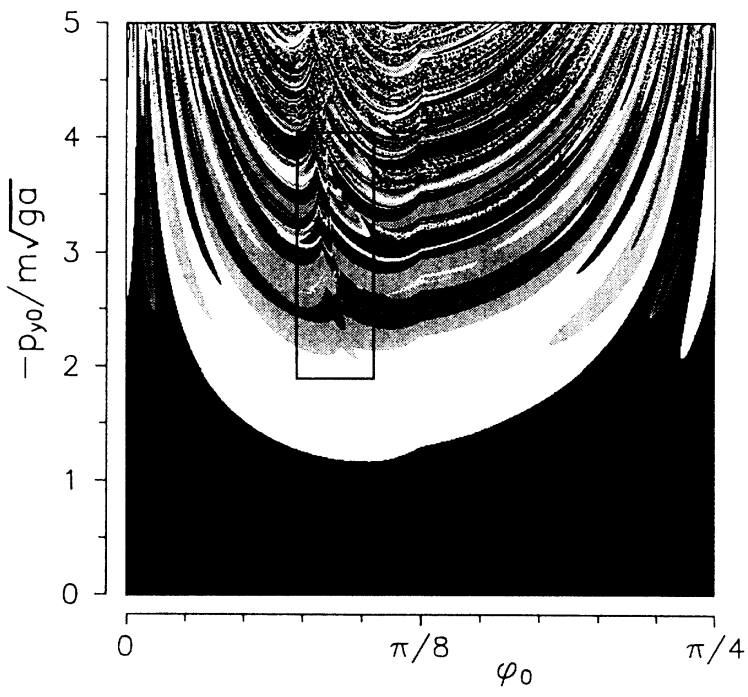

FIG. 5. Final outcome distribution for the case in which the die is started with an initial horizontal momentum $p_{x 0}=0$. Comparison with Fig. 4 shows that the basic structure in the outcome distribution is determined by the first few impacts. The later impacts introduce a good deal of fine structure. Nonetheless, one can still find regions where the initial condition can be varied significantly without changing the outcome.

Figure 6 shows an enlarged version of part of Fig. 5. The purpose of this figure is to illustrate the discontinuity in the final outcome distribution existing along the borderline between short and long flights after the first collision. Along this discontinuity, small changes in the initial conditions can change the outcome by more than 1 , i.e., for instance, from 1 to 3 . Along most of the other basic boundaries, the outcome only changes from one side of the die to a neighboring side.

Figures 7 and 8 show the final outcome distributions

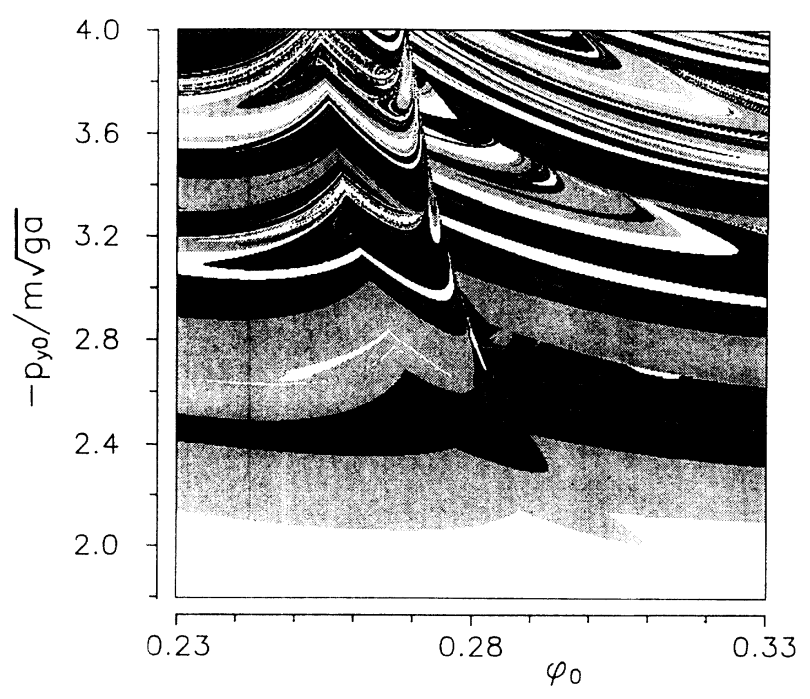

FIG. 6. Detail of Fig. 5 illustrating the fine structure which separates flat and nonflat collisions in the first impact. This fine structure is not yet fully understood. 


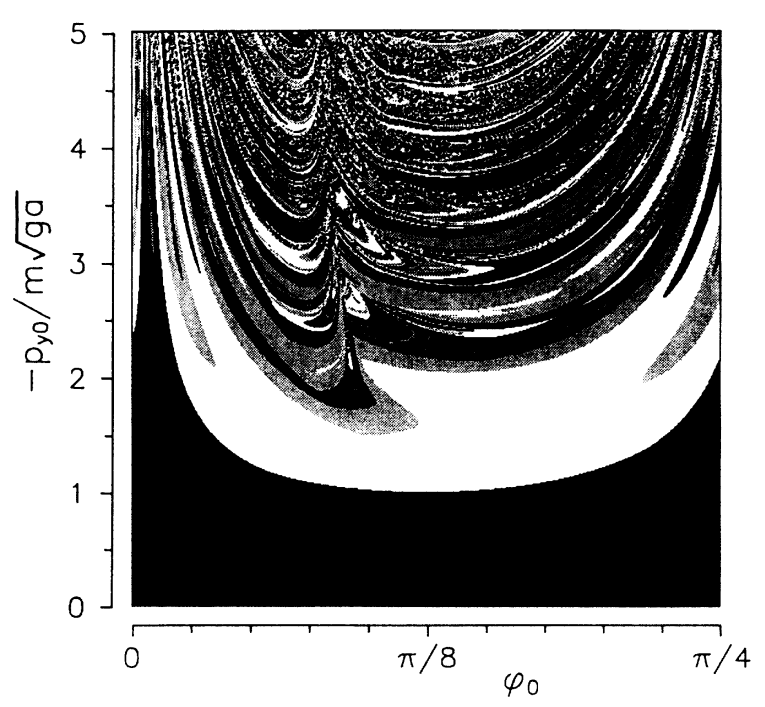

FIG. 7. Final outcome distributions for the case where the die is started with an initial momentum in the positive $x$ direction equal to $10 \%$ of the maximum, initial vertical momentum in the plot. The outcome distribution is deformed relative to the distribution observed in Fig. 5 for vanishing, horizontal momentum in the first impact.

for two cases in which the die is started with a finite initial momentum in the horizontal direction. In both cases, the numerical value of $p_{x 0}$ has been chosen to be $10 \%$ of the maximum initial vertical momentum in the considered outcome distribution. For Figure $7, p_{x 0}$ is in the positive $x$ direction, enforcing the rotation gained by the die in the first collision. For Fig. $8, p_{x 0}$ is negative and counteracts the rotation gained in the first collision. By comparison with Fig. 5, the presence of an initial horizontal momentum is seen to give rise to a distortion of the outcome distribution. Most of the basic features of this distribution remain unchanged, however.

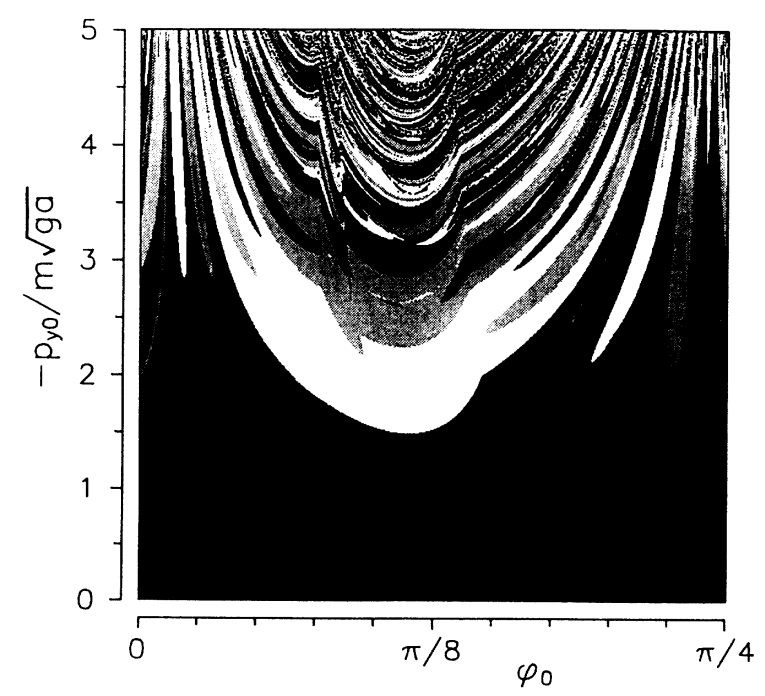

FIG. 8. Final outcome distribution for the case where the die is started with an initial momentum in the negative $x$ direction equal to $10 \%$ of the maximum, initial vertical momentum in the plot.

\section{PREIMAge Volume AND MIXING CALCULATIONS}

Let us hereafter turn our attention towards the problem of measuring the sensitivity of the final outcome with respect to changes in the initial conditions. This problem was also touched upon by Vulovic and Prange, ${ }^{5}$ who noted that for any point in a given basin of attraction a maximal variation in the initial conditions exists, which allows the point to remain in the same basin. This shortest linear extension was then used as a measure of the local uncertainty. In the present section we shall try to introduce somewhat more refined measures, which account both for the possibility of having more than two outcomes, and for the local distribution of outcomes.

First we shall consider the cross measure of basin area $A_{S}$. This measure is defined as the product $\Delta p_{y 0} \Delta \varphi_{0}$ of the ranges in momentum and orientation around a given initial condition within which the outcome remains the same. In contrast to a measure based on the linear distance to the nearest basin boundary, the cross measure recognizes the different dimensions of the two directions in the space of initial conditions. In the numerical calculations of $A_{S}$ we have normalized $\Delta p_{y 0}$ and $\Delta \varphi_{0}$ by division by $m \sqrt{g a}$ and $\pi / 4$, respectively. In this way, the maximum values for $A_{S}$ become of the order of 3 , namely a factor of 1 for small values of $p_{y 0}$ where the outcome distribution is independent of $\varphi_{0}$ times a factor of the order of 3 for those particular values of $\varphi_{0}$ where the outcome is independent of $p_{y 0}$ up to the highest values considered.

Figure 9 shows the variation of the cross measure of basin area with the initial conditions $\varphi_{0}$ and $p_{y 0}$ for the case where $p_{x 0}=0$. We have applied a 16 grade, logarithmic grey-tone scale with white denoting cross areas exceeding 2.2 and black denoting areas of less than $1.5 \times 10^{-5}$. Below a normalized initial momentum of

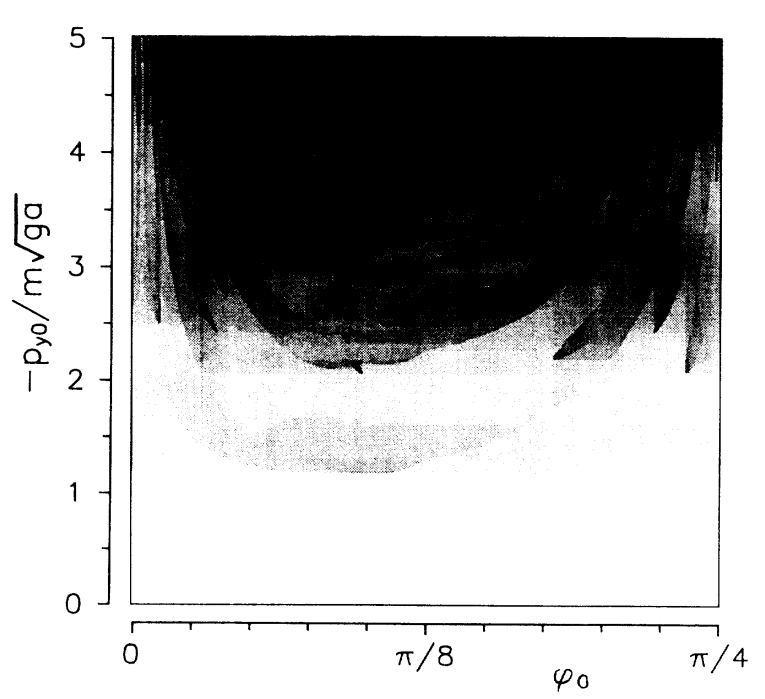

FIG. 9. Variation of the cross basin area $A_{S}$ with the initial conditions $\varphi_{0}$ and $p_{y 0}$ for $p_{x 0}=0$. The 16 grade grey-tone code is logarithmic with white denoting normalized cross areas exceeding 2.2 and black denoting areas less than $1.5 \times 10^{-5}$. 
$p_{y 0} / m \sqrt{g a} \cong-1.1$, the outcome is independent of $\varphi_{0}$ within the considered range of initial orientations. Close to $\varphi_{0}=0$ and $\pi / 4$ there are initial orientations where the outcome remains the same, even if the normalized initial momentum becomes larger than 2 . These are the regions where we find cross areas in excess of 2.2. In the upper part of Fig. 9, the cross areas are much smaller. However, as previously noted, one can still find regions where the initial conditions can be changed significantly without changing the outcome. Also, it is interesting to note how the cross area emphasizes the narrow tips in the fine structure of the outcome distribution.

A definition of mixing properties is well established for area-preserving maps. ${ }^{13}$ It expresses the idea of asymptotic measure invariance of map iterates on arbitrarily chosen subsets and their intersections. In the context of die tossing, the idea of a measure corresponds to the relative contents of different outcomes in a chosen subset of initial condition space. The mixing property may then be related to the uniformity of outcome distribution in all subsets. In that sense the final outcome map can never be fully mixing. Therefore we propose a local measure of mixing.

Let us choose a fixed neighborhood of a point in the space of initial conditions. For such a frame $\Gamma$, which is taken to contain a finite number of data points, we define a deviation from uniform distribution of the $i$ th outcome as

$$
d(\Gamma, i)=\left(\frac{c(\Gamma, i)}{n(\Gamma)}-\frac{1}{4}\right)^{2},
$$

where $c(\Gamma, i)$ is the number of frame points with outcome $i$, and $n(\Gamma)$ is the total number of points in the frame. The frame mixing coefficient $m$ is defined as

$$
m(\Gamma)=1-\frac{4}{3} \sum_{i=1}^{4} d(\Gamma, i)
$$

This definition is insensitive to the distribution of outcomes within the frame. In particular, it will not reflect the occurrence of larger connected subsets with the same outcome. Therefore we average over connected subframes $\gamma_{j}$ of a given size $k$ to obtain the local mixing factor

$$
\mathcal{F}(\Gamma, k)=\frac{\sum_{j} m\left(\gamma_{j}\right)}{N(\Gamma, k)}, \quad \gamma_{j} \subset \Gamma
$$

where the summation runs over the chosen connected subframes of size $k$, and $N(\Gamma, k)$ is the number of such subframes in $\Gamma$. To avoid a dependence of subframe size, we introduce the cumulative local mixing factor

$$
\mathcal{F}_{\text {cum }}=\prod_{l=1}^{l_{\max }} \mathcal{F}\left(\Gamma, k_{l}\right)
$$

where $k_{l}$ is a predefined sequence of subframe sizes.

It is of interest to relate the mixing measure defined in this way to randomness. We first observe that a homogeneous distribution (or a distribution with basins of attraction much larger than the frame size) will produce a

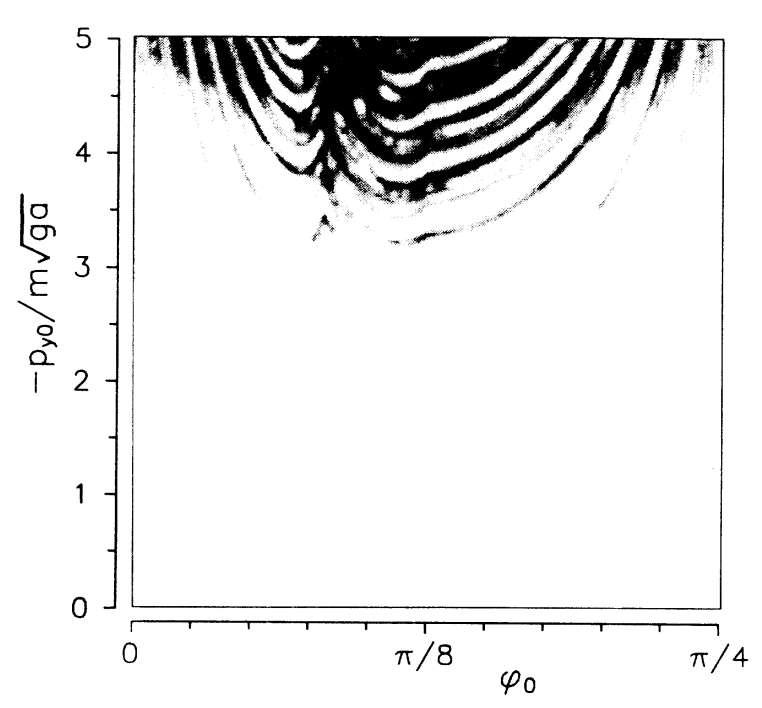

FIG. 10. Variation of the cumulative local mixing factor $\mathcal{F}_{\text {cum }}$ with the initial conditions. The 16 grade grey-tone code applied in this figure is linear with white denoting mixing factors less than $5 \times 10^{-5}$ and black denoting factors in excess of 0.6126 .

cumulative local mixing factor of 0 . It can easily be shown that large (i.e., close to 1) values of $\mathscr{F}_{\text {cum }}$ may be achieved for maps maximizing the factor in the same manner for all frames. This kind of mapping will exhibit a high degree of regularity in the form, for instance, of cyclic sequences. A random outcome map gives intermediate $\mathscr{F}_{\text {cum }}$ values (about 0.5 ) with a relative low variance as long as the frame size is reasonable.

The following calculations of $\mathscr{F}_{\text {cum }}$ were performed on the standard $\left(\varphi_{0}, p_{y 0}\right)$ space with $640 \times 480$ grid points. We applied a quadratic progression of subframe sizes slightly modified to give numbers divisible by 4 . The

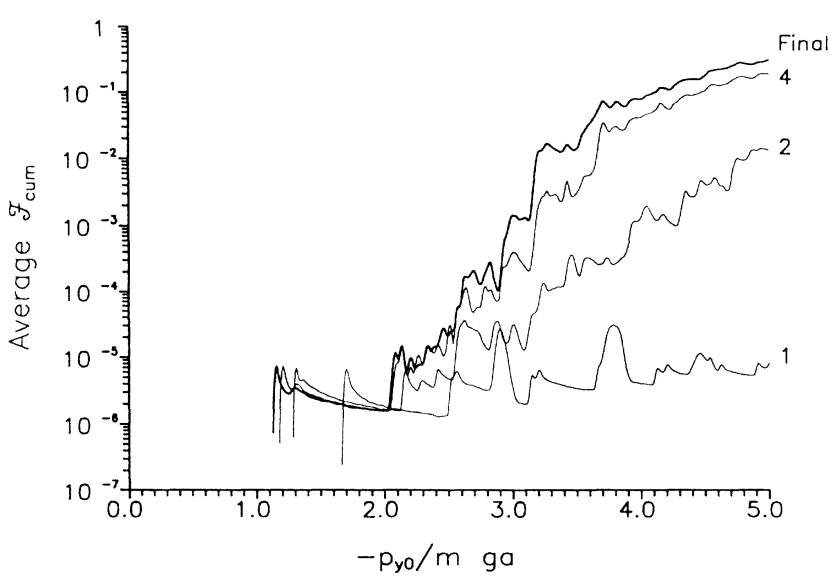

FIG. 11. Variation of the cumulative local mixing factor with the normalized initial momentum after one, two, and four iterations of the combined collision-flight maps. Also shown is the corresponding variation for the final outcome distribution. All curves on this figure represent averages over the initial orientation of the die. After the first four iterations of $F \circ C$, little change in the average $\mathscr{F}_{\text {cum }}$ occurs. 


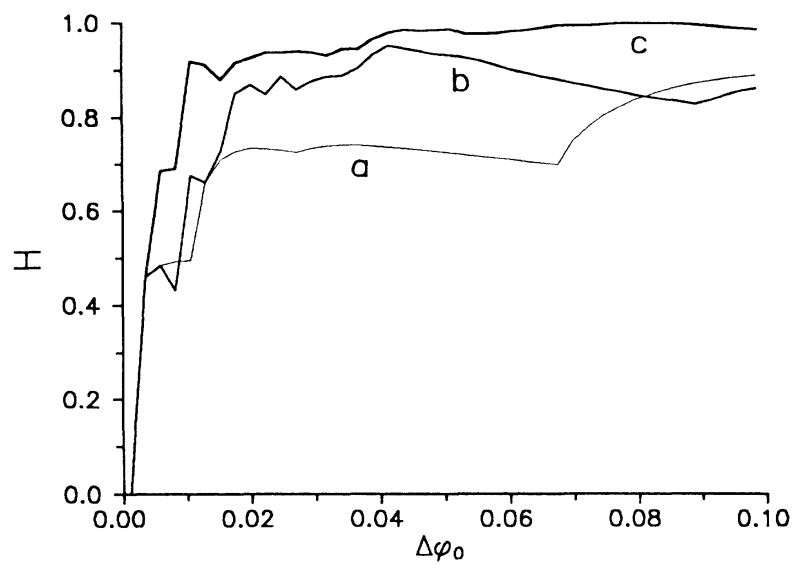

FIG. 12. Variation in the entropy function $H\left(\Delta \varphi_{0}\right)$ as the initial orientation interval of the die is gradually changed for three different values of the initial vertical momentum, $p_{y 0} / m \sqrt{g a}=3,4$, and 5 , for (a), (b), and (c), respectively. In all cases $\varphi_{0}=\pi / 8$.

chosen progression is $4,8,16,24,36,48,64,80$, and 100 grid points. As shown in Fig. 10, the cumulative local mixing factor depends heavily on both $p_{y 0}$ and $\varphi_{0}$. The 16 grade grey-tone code applied in this figure is linear with white denoting mixing factors less than $5 \times 10^{-5}$, and black denoting mixing factors in excess of 0.6126 . The variation in $\mathscr{F}_{\text {cum }}$ clearly reflects the curtainlike structure of the outcome distribution itself. Compared with the cross basin area, the mixing factor gives more emphasis to borderlines between outcomes and less emphasis to narrow tips. In Fig. 11, we have compared the variation of $\mathcal{F}_{\text {cum }}$ obtained for the final outcome distribution with the variations observed after one, two, and four collision-flight pairs. Within the investigated range of initial energies, most of the mixing is found to be produced during the first four collisions.

As a third example of a measure of local uncertainty we have calculated the variation in the entropy of the outcome distribution

$$
H(d)=-\sum_{i=1}^{n} \frac{\mu_{i}(d)}{\mu(d)} \log _{n} \frac{\mu_{i}(d)}{\mu(d)}
$$

for a neighborhood of a particular point in the space of initial conditions as the neighborhood is expanded by gradually allowing a larger uncertainty in the orientation of the die. A similar calculation can be performed for changes in the vertical momentum. $n=4$ is here the number of possible outcomes, and $d=\Delta \varphi_{0}$ is the diameter of the considered neighborhood in the space of initial conditions. By $\mu(d)$ we denote the measure of the whole neighborhood (i.e., the number of the data points in $\Delta \varphi_{0}$ ) and by $\mu_{i}(d)$ the measure of its intersection with the $i$ th outcome.

It is well known that the difference between the actual entropy and the maximal value for a given outcome distribution can be used to characterize the deviation from a uniform distribution. For a random sequence, $H(d)$ approaches 1 , as the number of data points become large. For sufficiently high initial energies, the same result is ex- pected for the die. The interesting aspect of the problem is therefore the manner in which this approach occurs. As long as the number of data points is sufficient to resolve the structure of the outcome distribution, $H(d)$ is independent of the density of data points. As in the case with the mixing factor, this measure integrates information associated with the interleafed basins of attraction with the corresponding variation in outcome.

Figure 12 shows a calculation of $H\left(\Delta \varphi_{0}\right)$ for three points in the space of initial conditions with $p_{y 0} / m \sqrt{g a}=3,4$, and 5 , respectively. In all cases $\varphi_{0}=\pi / 8$. For all curves there is a range around $\Delta \varphi_{0}=0$ in which $H\left(\Delta \varphi_{0}\right)=0$. This is the range in which the outcome remains constant. $H\left(\Delta \varphi_{0}\right)$ thereafter increases towards a saturation value for large $\Delta \varphi_{0}$. The rate at which this increase occurs measures the uncertainty in the local outcome distribution.

\section{CONCLUSION}

In a rather surprising manner, the development of nonlinear dynamics has disclosed a number of misconceptions in our traditional understanding of determinism. In particular, it has been shown that the notion of predictability, according to which the trajectory of a system can be precisely calculated if one knows the equations of motion and the initial conditions, is related to textbook examples of integrable systems. This predictability does not extend to nonlinear, conservative systems in general. ${ }^{14}$ Dissipative systems can also show unpredictability provided that their motion is sustained by externally supplied energy. Although traditionally considered as unpredictable, die tossing does not belong to any of these categories. Accordingly, the toss of a die only shows a finite sensitivity to the initial conditions.

Nonlinear dynamics has also provided a variety of new approaches to complex dynamic problems. One such approach involves the application of discrete maps to represent continuous dynamical systems. Other examples of techniques and concepts derived from nonlinear dynamics are those of symbolic dynamics and selfsimilarity. These will be applied in a forthcoming publication to address the problem of the asymptotic properties of die tossing for high initial energies. In particular, we shall calculate how rapidly the basins of attraction shrink as the initial energy becomes very large.

In the present paper we have applied dissipative mapping to study the distribution of outcomes for a die toss. This technique appears well suited for a number of problems involving multiple collisions. A simple example could be the classical pinball (or Galton) machine, where balls fall between horizontal pins to produce a binomial distribution at the bottom. Traditionally, this problem has also been treated in a stochastic manner, although clearly the equations of motion are derivable from Newton's laws. A revised treatment would consider the trajectories of the balls in a fully deterministic manner. The stochasticity then identifies itself as arising through amplification of uncertainties in the initial conditions, when the balls enter the machine. Of course, this uncertainty may again be related to the forces that the balls 
have experienced before they entered the machine. In this manner, one can push the borderline between predictable and unpredictable behavior backwards.

Now, if die tossing and the motion of a ball in a pinball machine are predictable, what is then random? For the moment we think that the best answer to this question is to notice that the word random is used to characterize events (or processes) which, for one reason or another, we cannot calculate (or control). Even if a process in principle is deterministic, we may consider it as random if the complexity involved is so high that we cannot relate cause and effect in detail. In this sense, die tossing is still random. The predictability shows up, only if the initial energy is small or, if the tossing is performed by a very accurate machine.

From a practical point of view, the main thing is that the mapping technique allows us to deal with a number of problems which hitherto have been considered untractable. In a more general version, the same approach can be applied to systems which are integrable except for certain abrupt shifts associated, for instance, with logical elements.

\section{ACKNOWLEDGMENTS}

We would like to thank Kunihiko Kaneko for suggesting the application of the entropy measure. Ellen Buchhave is acknowledged for a number of stimulating discussions.
*Permanent address: Institute of Automatics, University of Mining and Metallurgy, Cracow, Poland.

${ }^{1}$ H. G. Schuster, Deterministic Chaos (VCH Verlagsgesellschaft, Weinheim, 1989).

${ }^{2}$ P. Bergé, Y. Pomeau, and C. Vidal, Order within Chaos (Wiley, New York, 1984).

3J. A. Yorke and E. D. Yorke, J. Stat. Phys. 21, 269 (1979).

${ }^{4}$ C. Grebogi, E. Ott, and J. A. Yorke, Phys. Rev. Lett. 50, 935 (1983).

${ }^{5}$ V. Z. Vulovic and R. E. Prange, Phys. Rev. A 33, 576 (1986).

${ }^{6}$ C. Knudsen, R. Feldberg, M. Hindsholm, and E. Mosekilde, Int. Assoc. Math. Comput. Simulation Ann. Comput. Appl. Math. 1, 61 (1989).

${ }^{7}$ E. Mosekilde, C. Knudsen, R. Feldberg, and M. Hindsholm, Proceeding of the Fourth International IPSO Conference on Irreversible Processes and Self-Organization, Rostock, German Democratic Republic, 1989 (Teubner Verlagsgesellschaft, Leipzig, 1989); Teubner-Texte zur Physik 23: Irreversible Pro- cesses and Self-Organization, edited by W. Ebeling and H. U1bricht (Teubner Verlagsgesellschaft, Leipzig, 1989), pp. 84-97.

${ }^{8}$ C. Grebogi, S. McDonald, E. Ott, and J. A. Yorke, Phys. Lett. 99A 415 (1983).

${ }^{9}$ S. W. Groesberg, Advanced Mechanics (Wiley, New York, 1968).

${ }^{10} \mathrm{C}$. Knudsen and R. Feldberg (unpublished).

${ }^{11}$ S. McDonald, C. Grebogi, E. Ott, and J. A. Yorke, Phys. Lett. 107A, 51 (1985).

${ }^{12}$ C. Grebogi, E. Kostelich, E. Ott, and J. A. Yorke, Physica D 25, 347 (1987).

${ }^{13}$ A. Lasota and M. C. Mackey, Probabilistic Properties of Deterministic Systems (Cambridge University Press, Cambridge, 1985).

${ }^{14}$ R. H. G. Helleman, in Fundam. Prob. Stat. Mech. 5, 165 (1980). 


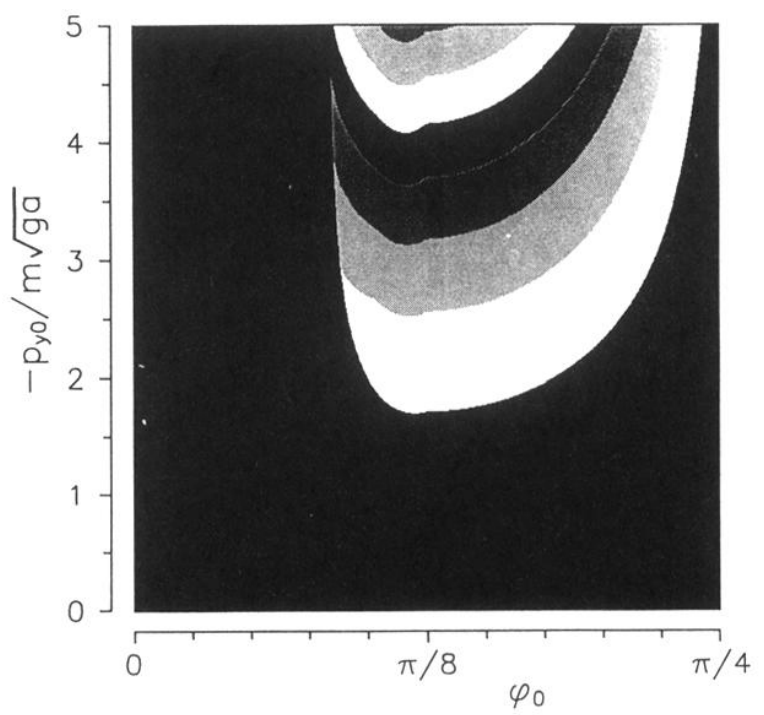

FIG. 1. Outcome distribution after one iteration of the combined collision-flight map $F \circ C$. The horizontal axis represents the orientation of the die before the first collision, and the vertical axis shows the normalized initial momentum $p_{y 0} / m \sqrt{g a}$. The die is assumed to fall vertically from an altitude $h_{0}=p_{y 0}^{2} / 2 m^{2} g+(a / \sqrt{2}) \cos \left(\varphi_{0}-\pi / 4\right)$. With the applied color code, black represents 1 , dark grey 2 , light grey 3 , and white 4 . The lack of structure on the left-hand side of the figure is due to short flights. Note the discontinuity in the outcome variation across the borderline between short and long flights. 


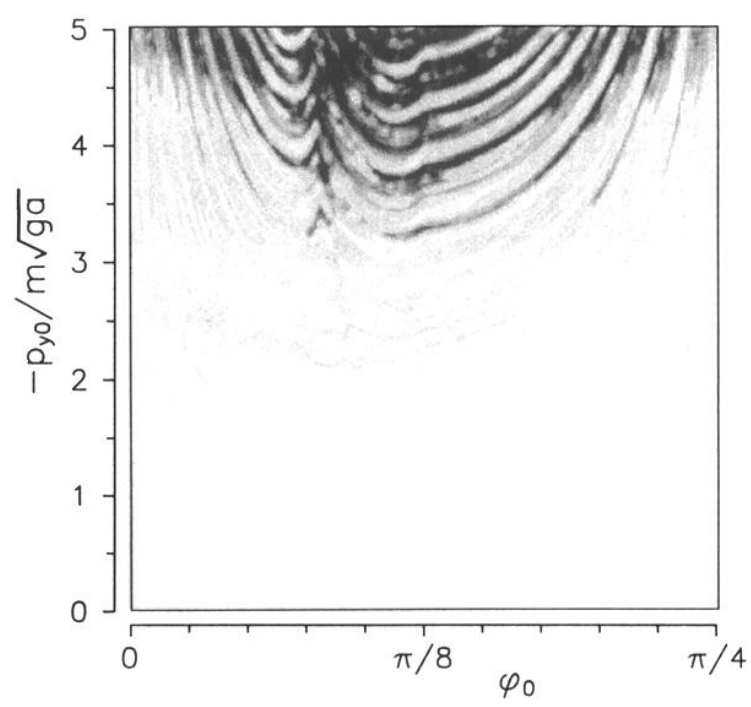

FIG. 10. Variation of the cumulative local mixing factor $\mathcal{F}_{\text {cum }}$ with the initial conditions. The 16 grade grey-tone code applied in this figure is linear with white denoting mixing factors less than $5 \times 10^{-5}$ and black denoting factors in excess of 0.6126 . 


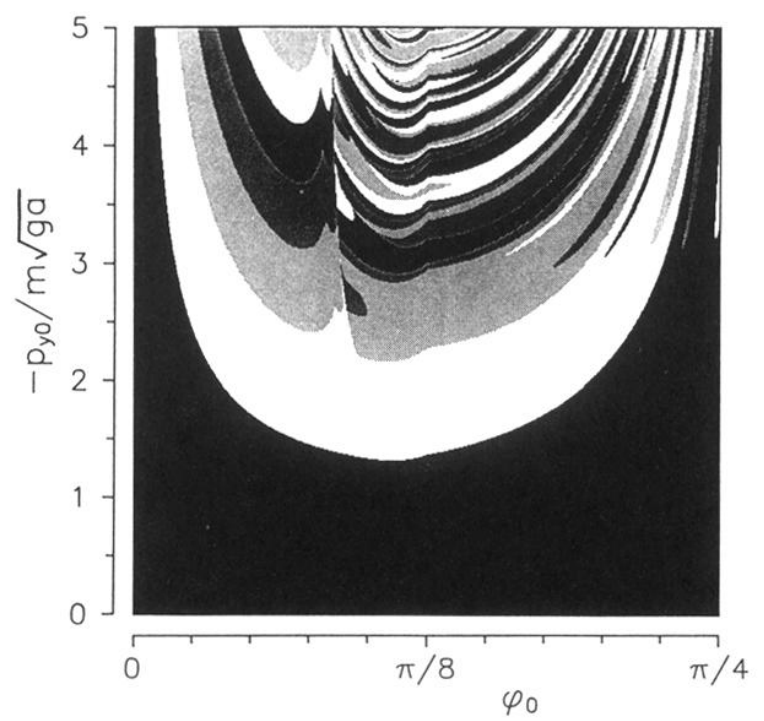

FIG. 2. Outcome distribution after two iterations of $F \circ C$. The second collision gives rise to a continuation of the outcome distribution produced by the first impact into the left-hand side of the space of initial conditions. Broadly speaking, this continuation has the form of a slightly deformed mirror image of the structure on the right-hand side of Fig. 1. On the righthand side of this figure, additional structure has now emerged in each of the previous bands of constant outcome. 

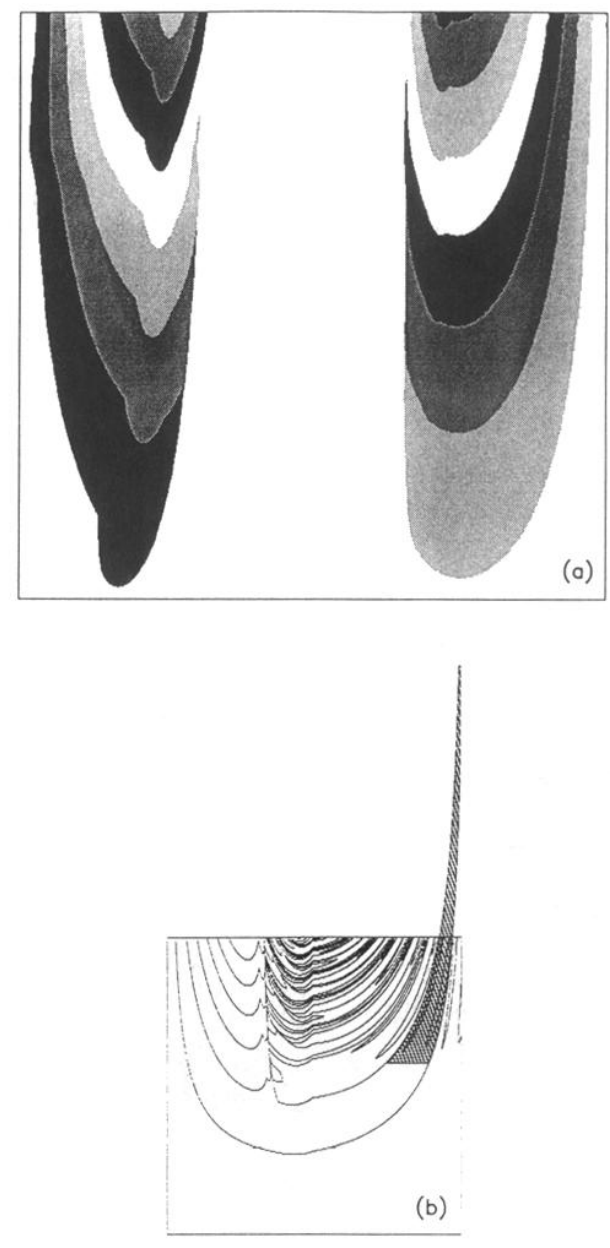

FIG. 3. Amplification and transformation of one of the bands in Fig. 2 show that the substructure produced by the second impact contains a double version of the structure observed in Fig. 1 after the first collision. This is expressive of the recurrent properties of the mapping process. The sketch in (b) shows the part of Fig. 2 that has been transformed into (a). 


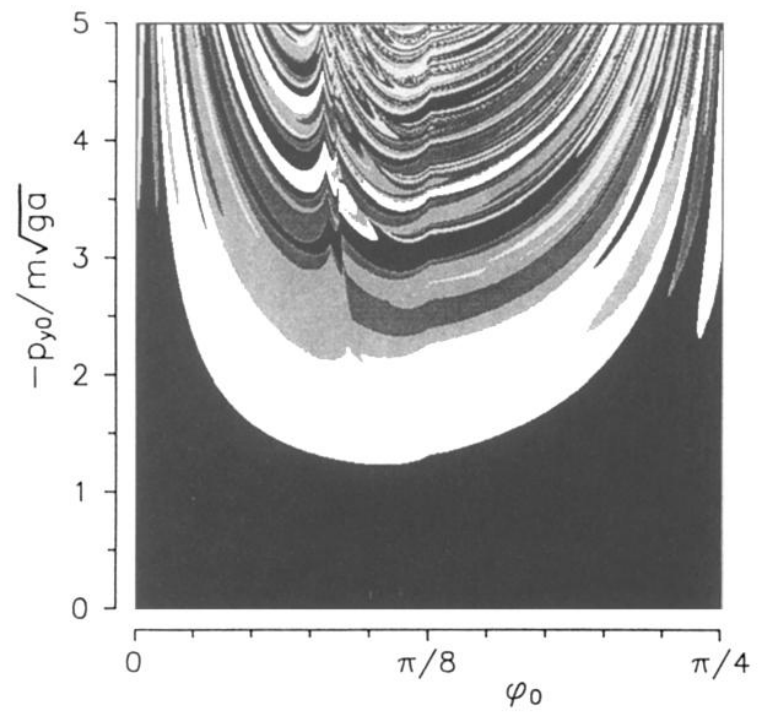

FIG. 4. Outcome distribution after three iterations of $F \circ C$. The substructure observed on the right-hand side of Fig. 2 now appears on the left-hand side of this figure. On the right-hand side, additional substructure has appeared, as many of the areas of constant outcome in Fig. 2 have been divided into several smaller ones. Again there is a certain self-similarity in the process. 


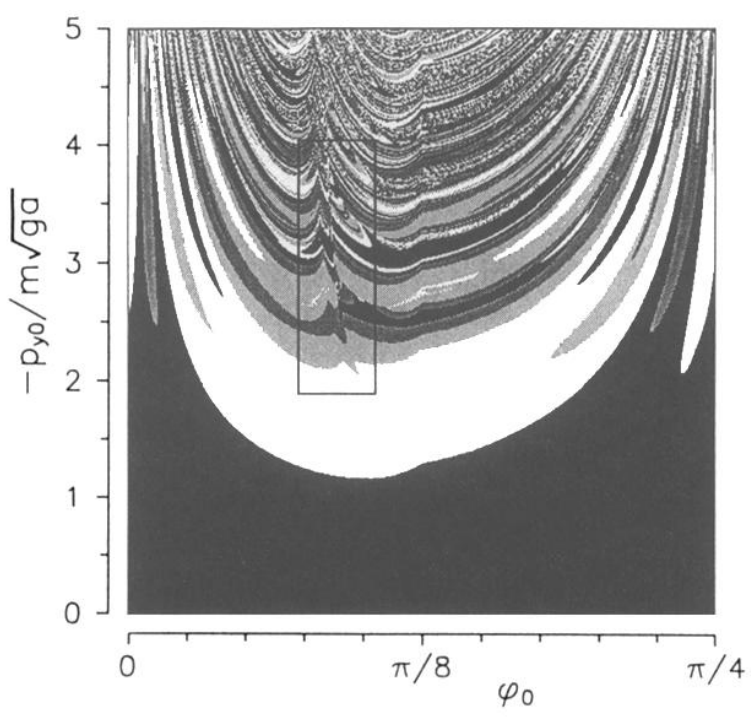

FIG. 5. Final outcome distribution for the case in which the die is started with an initial horizontal momentum $p_{x 0}=0$. Comparison with Fig. 4 shows that the basic structure in the outcome distribution is determined by the first few impacts. The later impacts introduce a good deal of fine structure. Nonetheless, one can still find regions where the initial condition can be varied significantly without changing the outcome. 


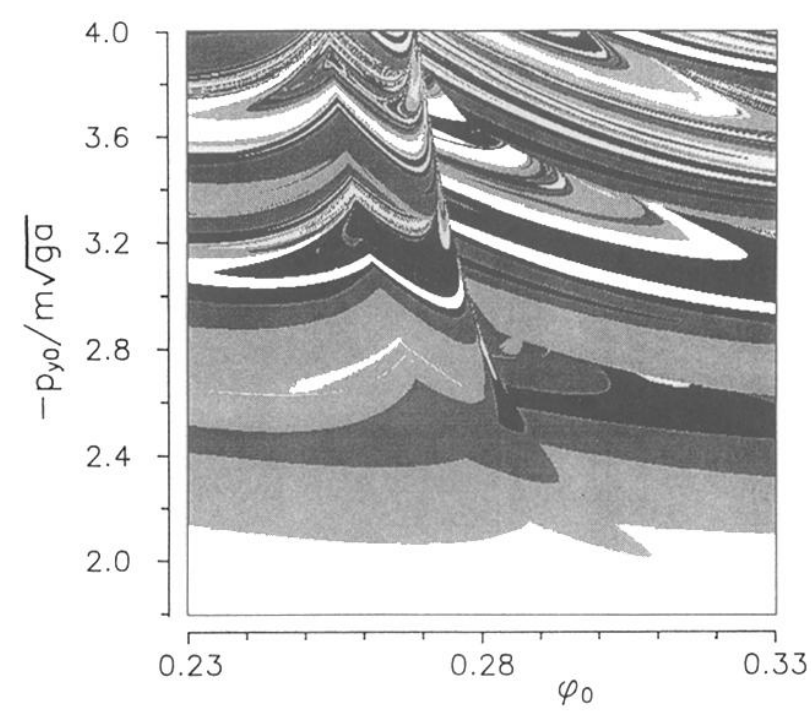

FIG. 6. Detail of Fig. 5 illustrating the fine structure which separates flat and nonflat collisions in the first impact. This fine structure is not yet fully understood. 


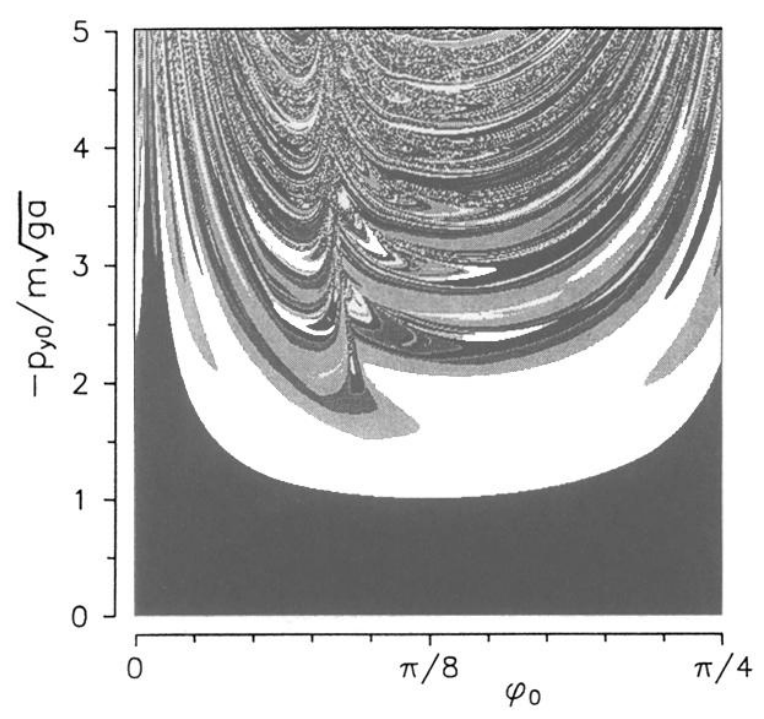

FIG. 7. Final outcome distributions for the case where the die is started with an initial momentum in the positive $x$ direction equal to $10 \%$ of the maximum, initial vertical momentum in the plot. The outcome distribution is deformed relative to the distribution observed in Fig. 5 for vanishing, horizontal momentum in the first impact. 


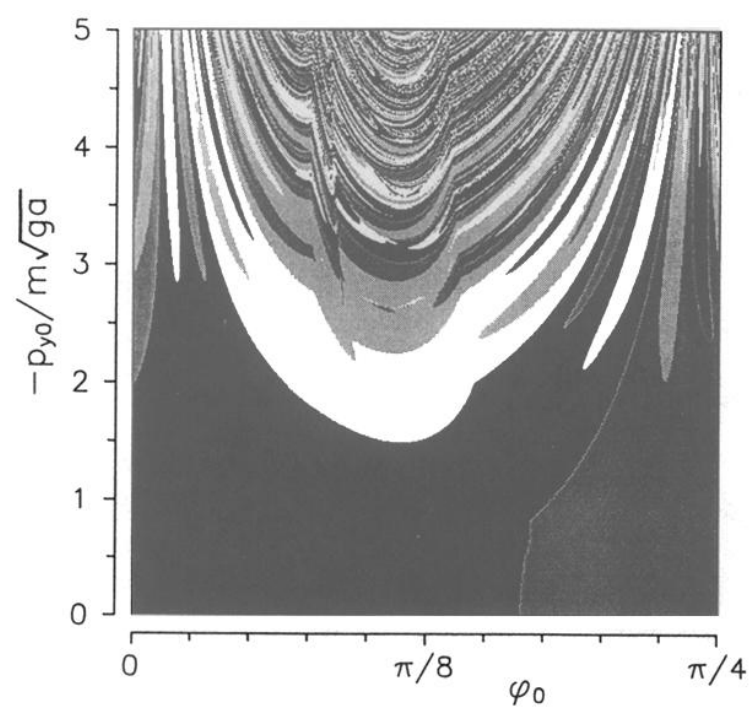

FIG. 8. Final outcome distribution for the case where the die is started with an initial momentum in the negative $x$ direction equal to $10 \%$ of the maximum, initial vertical momentum in the plot. 


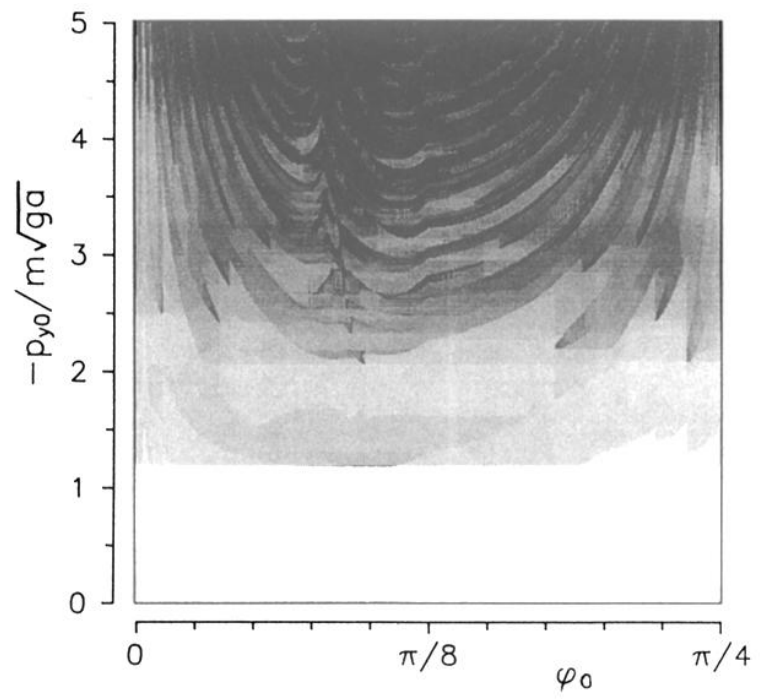

FIG. 9. Variation of the cross basin area $A_{S}$ with the initial conditions $\varphi_{0}$ and $p_{y 0}$ for $p_{x 0}=0$. The 16 grade grey-tone code is logarithmic with white denoting normalized cross areas exceeding 2.2 and black denoting areas less than $1.5 \times 10^{-5}$. 